\title{
Homomorphisms of finitely generated Kleinian groups
}

\author{
Dedicated to Prof. Y. Kusunoki on his $60^{\text {th }}$ birthday
}

By

Makoto MASUMOTO

(Received March 1, 1983)

\section{Introduction.}

In this paper we will study about the neighborhoods of the identity homomorphism id in the space $\operatorname{Hom}(\Gamma, M \ddot{b} b)$ of homomorphisms of a finitely generated Kleinian group $\Gamma$. (For definitions, see §3, (1).)

Some elements of $\operatorname{Hom}(\Gamma, M \ddot{b} b)$ can be constructed by means of quasiconformal deformations or Schwarzian differential equations. Gardiner$\mathrm{Kra}$ [5] showed that all elements of $\operatorname{Hom}_{\Delta-p}(\Gamma, M \ddot{b} b)$ sufficiently close to id can be so obtained if $\Gamma$ is a b-group with an invariant (simply connected) component 4 . Here, we shall show that this is also true for any finitely generated Kleinian group. This is a corollary to Theorem 1 . (See Corollary 2 to Theorem 1 in §4.) As applications of this corollary, we shall give some theorems on quasi-stability.

In $\S 1$ the statement of Theorem 1 , the main result, will be given. We use the techniques of Gardiner-Kra[5] to prove Theorem 1. Its proof is somewhat long and will be completed in $\S 4$. In $\S 2$ we provide a theorem (Theorem 2) on cohomology, which will play an important role in the proof of Theorem 1. Theorem 2 can be proved by means of some results due to Ahlfors[1] and $\mathrm{Kra}[6] . \S 4$ is devoted to the proof of Theorem 1. At the end of this section, two corollaries will be given.

In the rest of the paper, we are interested in the solutions of certain Schwarzian differential equations. In $\S 5$ we will give a property of quasi-stable groups. In $\S 6$ we will give some necessary and sufficient conditions that a finitely generated function group should be quasi-stable. In this section, the results of $\mathrm{Kra}$ [7] and Maskit[12] play an essential role.

Finally, the author wishes to express his deepest gratitude to Prof. Y. Kusunoki for valuable suggestion and kind guidance. The author also thanks to Mr. H. Ohtake for his advice. 


\section{§1. Statement of Theorem 1.}

Let $\Gamma$ be any finitely generated (non-elementary) Kleinian group. (In this paper all Kleinian groups are assumed to be non-elementary.) Let $\Delta$ be an invariant union of components of $\Gamma$. We donote by $A(\Delta, \Gamma)$ the complex Banach space of all bounded holomorphic automorphic forms of weight $(-4)$ on $\Delta$ for $\Gamma . A(\Delta, \Gamma)$ is of finite dimension. $M(\Delta, \Gamma)$ will denote the complex Banach space (with the supremum norm) of all Beltrami differentials for $\Gamma$ that are equal to zero on $\hat{\boldsymbol{C}}-\Delta$. Its closed subspace $M_{c a n}(\Delta, \Gamma)$ consists of all elements $\mu \in M(\Delta, \Gamma)$ with the property that $\left.\lambda_{\Delta}^{2} \bar{\mu}\right|_{\Delta} \in A(\Delta, \Gamma)$, where $\lambda_{\Delta}(z)|d z|$ denotes the Poincaré metric on $\Delta$. The unit balls of $M(\Delta, \Gamma)$ and $M_{c a n}(\Delta, \Gamma)$ will be denoted by $M(\Delta, \Gamma)_{1}$ and $M_{c a n}(\Delta, \Gamma)_{1}$, respectively.

Let us now assume that $S=\Delta / \Gamma$ is a single Riemann surface. We remove the elliptic fixed points from $\Delta$ and denote the punctured open set by $\Delta_{0}$. We set $S_{0}=\Delta_{0} / \Gamma . \quad S-S_{0}$ consists of finitely many points $p_{1}, \ldots, p_{k}$ and the natural projection $\Delta \longrightarrow S$ is branched at each lift of $p_{j}$ with the multiplicity $r_{j}(j=1, \ldots, k)$. Fix $p_{0} \in S_{0}$ and form the fundamental group $\pi_{1}\left(S_{0}, p_{0}\right)$ of $S_{0}$ with the base point $p_{0}$. Choose simple loops $c_{j}$ from $p_{0}$ around $p_{j}(j=1, \ldots, k)$. Let $N$ be the normal subgroup of $\pi_{1}\left(S_{0}, p_{0}\right)$ generated by $\left\{c_{j}^{r_{j}{ }_{j}}\right\}_{j=1, \ldots, k}$. We denote the quotient group $\pi_{1}\left(S_{0}, p_{0}\right) / N$ by $G=G\left(\Delta, \Gamma ; p_{0}\right)$. The image of $c \in \pi_{1}\left(S_{0}, p_{0}\right)$ under the canonical surjection $\pi_{1}\left(S_{0}, p_{0}\right) \longrightarrow G$ is denoted by $c N$. $c N$ is a (right) coset modulo $N$.

Fix $\zeta_{0} \in \Delta_{0}$ with projection $p_{0}$. Let $\Delta_{0}^{\prime}$ be the component of $\Delta_{0}$ that contains $\zeta_{0}$, and set $\Gamma^{\prime}=\operatorname{stab}\left(\Delta_{0}^{\prime}\right)=\left\{\gamma \in \Gamma \mid \gamma\left(\Delta_{0}^{\prime}\right)=\Delta_{0}^{\prime}\right\}$. In familiar manner, $\Delta_{0}^{\prime}$ is a smooth covering surface of $S_{0}$ determined by a normal subgroup $N^{\prime}$ of $\pi_{1}\left(S_{0}, p_{0}\right)$, and there is an isomorphism $\tilde{\sigma}: \pi_{1}\left(S_{0}, p_{0}\right) / N^{\prime} \longrightarrow \Gamma^{\prime}$. Since $N$ is a subgroup of $N^{\prime}$, this isomorphism $\tilde{\sigma}$ induces a (surjective) homomorphism $\sigma=\sigma\left(\Delta, \Gamma ; \zeta_{0}\right): G \longrightarrow \Gamma^{\prime}$. Explicitly, the homomorphism $\sigma$ is defined as follows. A closed curve $c$ from $p_{0}$ (on $S_{0}$ ) lifts to a curve $c^{\prime}$ on $\Delta_{0}^{\prime}$ with the initial point $\zeta_{0}$. There is a unique element $r \in \Gamma^{\prime}$ such that $\gamma\left(\zeta_{0}\right)$ is the end point of $c^{\prime}$. Then, we define $\sigma(c N)=\gamma$.

Let $M o ̈ b$ denote the 3-dimensional complex Lie group of all Möbius transformations. Denote the set of all homomorphisms from $G$ into $M o ̈ b$ by $\operatorname{Hom}(G, M \ddot{o} b) . \operatorname{Hom}_{p}(G, M \ddot{o} b)$ is the set of all $\chi \in \operatorname{Hom}(G, M \ddot{o} b)$ such that $\operatorname{trace}^{2} \chi(c N)=4$ whenever $c$ is a simple loop around a puncture of $S$. Note that $\sigma \in \operatorname{Hom}_{p}(G, M \ddot{b} b) . G$ is generated by finitely many elements, say, by $\tilde{c}_{1} N, \ldots, \tilde{c}_{r} N$. Then one can $\operatorname{regard} \operatorname{Hom}(G, M \ddot{b} b)$ as a subset of $(M \ddot{o} b)^{r}$ by the correspondence $\operatorname{Hom}(G, M \ddot{o} b) \ni \chi \longleftrightarrow\left(\chi\left(\tilde{c}_{1} N\right), \ldots, \chi\left(\tilde{c}_{r} N\right)\right)$ $\in(M \ddot{o} b)^{r}$, and make it a topological space. This topology does not depend on a particular choice of a system of generators of $G$.

We are now ready to state the following 
Theorem 1. Let $\Gamma$ be a finitely generated (non-elementary) Kleinian group and let $\Delta$ be an invariant union of components of $\Gamma$. Assume that $\Delta / \Gamma$ is a single Riemann surface. Fix $\zeta_{0} \in \Delta-\{$ elliptic fixed points $\}$ and let $p_{0} \in \Delta / \Gamma$ be its projection. Set $G=G\left(\Delta, \Gamma ; p_{0}\right)$ and $\sigma=\sigma\left(\Delta, \Gamma ; \zeta_{0}\right)$. Then $\operatorname{Hom}_{p}(G, M o ̈ b)$ is a complex manifold in a neighborhood of $\sigma$. More precisely, there is a holomorphic map $f: M \ddot{b} b \times A(\Delta, \Gamma) \times M_{c a n}(\Delta, \Gamma)_{1} \longrightarrow \operatorname{Hom}_{p}(G, M o ̈ b)$ with $f(e, 0,0)=\sigma$ such that $f$ induces a biholomorphic map between a neighborhood of $(e, 0,0)$ and a neighborhood of $\sigma$, where $e$ denotes the unit element of $M o ̈ b$.

To prove Theorem 1, we need some auxiliary resulsts. The proof of Theorem 1, together with its corollaries, will appear in $\S 4$.

In the following, to avoid confusion, we shall denote by $e$ the unit element of the group $M \ddot{o} b$, and by id the identity homomorphism.

\section{§2. A theorem on cohomology of $G\left(\Delta, \Gamma ; p_{0}\right)$.}

The purpose of this section is to prove Theorem 2, which will play an important role in the proof of Theorem 1 .

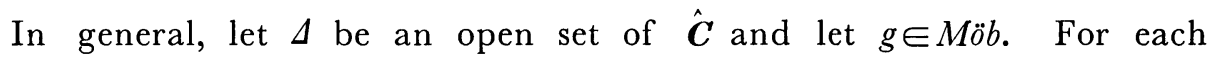
function $\varphi$ on $g(\Delta)$, we define a function $g_{p, q}^{*} \varphi$ on $\Delta$ by $g_{p, q}^{*} \varphi(z)=$ $\varphi(g(z)) g^{\prime}(z)^{p}{\overline{g^{\prime}(z)}}^{q}(z \in \Delta)$, where $p$ and $q$ are integers. $g_{p, 0}^{*}$ will be abbreviated to $g_{p}^{*}$.

First of all, we shall give an outline of the cohomology theory of Kleinian groups due to Kra. For details, see Kra [9, Chapter V].

Denote by $\Pi$ the vector space of polynomials in one complex variable of degree at most 2. $M \ddot{b} b$ acts on the right on $\Pi$ via $\Pi \times M \ddot{0} b \ni(v, g)$ $\longmapsto v \cdot g=g_{-1}^{*} v \in \Pi$.

Let $\Gamma$ be a finitely generated Kleinian group and let $\Delta$ be an invariant union of components of $\Gamma$. Since $\Gamma$ acts on $\Pi$, one can define the cohomology groups. We will denote by $H^{1}(\Gamma, \Pi)$ and $P H_{\Delta}^{1}(\Gamma, \Pi)$ the (first) cohomology space and the space of $\Delta$-parabolic cohomology classes, respectively. We also denote by $Z^{1}(\Gamma, \Pi)$ and $P Z_{\Delta}^{1}(\Gamma, \Pi)$ the space of cocycles and the space of $\Delta$-parabolic cocycles, respectively.

Let $\beta^{*}=\beta_{\Gamma, \Delta}^{*}: M_{c a n}(\Delta, \Gamma) \longrightarrow P H_{\Delta}^{1}(\Gamma, \Pi)$ be the Bers map (cf. [9, V, Theorem 2.4]). It is known that $\beta^{*}$ is injective.

The space of bounded Eichler integrals (modulo $\Pi$ ) of order $(-1)$ (on $\Delta$ for $\Gamma$ ) will be denoted by $E_{b}(\Delta, \Gamma)$. There is a canonical injective linear mapping (called the period map) $\alpha=\alpha_{\Gamma \cdot \Delta}: E_{b}(\Delta, \Gamma) \longrightarrow P H_{\Delta}^{1}(\Gamma, \Pi)$ (cf. [9, V, Theorem 4.2]).

$\mathrm{Kra}$ [6] proved that there is a direct decomposition

$$
P H_{\Delta}^{1}(\Gamma, \Pi)=\alpha\left(E_{b}(\Delta, \Gamma)\right)+\beta^{*}\left(M_{c a n}(\Delta, \Gamma)\right)
$$

(cf. [9, V, Corollary 1 to Theorem 5. 1]). 
Next, we assume that $S=\Delta / \Gamma$ is a single Riemann surface, and use the same notation as in $\S 1$. $G$ acts on the right on $\Pi$ by $\Pi \times G \ni(v, c N) \longmapsto$ $v \cdot \sigma(c N) \in \Pi$. We denote by $Z^{1}(G, \Pi)$ the space of cocycles, and by $H^{1}(G, \Pi)$ the cohomology space. A cocycle $q \in Z^{1}(G, \Pi)$ is called parabolic if $\left.q\right|_{<c N\rangle}$ is a coboundary for the cyclic subgroup $\langle c N\rangle$ whenever $c \in \pi_{1}\left(S_{0}, p_{0}\right)$ is a simple loop around a puncture of $S$. We denote by $P Z^{1}(G, \Pi)$ the subspace consisting of all parabolic cocycles, and by $P H^{1}(G, \Pi)$ the space of all parabolic cohomology classes.

There is a canonical linear map $J_{0}: Z^{1}(\Gamma, \Pi) \longrightarrow Z^{1}(G, \Pi)$ defined by $J_{0}(q)(c N)=q(\sigma(c N)) \quad\left(q \in Z^{1}(\Gamma, \Pi), c N \in G\right)$. $J_{0}$ induces a linear map $J: P H_{\Delta}^{1}(\Gamma, \Pi) \longrightarrow P H^{1}(G, \Pi)$. Let $\Delta^{\prime}$ be the component of $\Delta$ that contains $\zeta_{0}$, and set $\Gamma^{\prime}=\operatorname{stab}\left(\Delta^{\prime}\right)$. Since $\sigma(G)=\Gamma^{\prime}, J_{0}(q)=0$ if and only if $q(\gamma)=0$ for all $r \in \Gamma^{\prime}$.

Lemma 1. $\operatorname{dim} J \circ \beta^{*}\left(M_{c a n}(\Delta, \Gamma)\right)=\operatorname{dim} A(\Delta, \Gamma)$.

Proof. It suffices to prove that $J \circ \beta^{*}: M_{c a n}(\Delta, \Gamma) \longrightarrow P H^{1}(G, \Pi)$ is injective. Suppose that $J \circ \beta^{*}(\mu)=0$ with $\mu \in M_{c a n}(\Delta, \Gamma)$. For $z \in C$ set

and

$$
\begin{aligned}
& h(z)=\frac{z(z-1)}{2 \pi i} \iint_{c} \frac{\mu(\zeta)}{\zeta(\zeta-1)(\zeta-z)} d \zeta \wedge d \bar{\zeta}, \\
& h_{1}(z)=\frac{z(z-1)}{2 \pi i} \iint_{c} \frac{\mu(\zeta) \chi(\zeta)}{\zeta(\zeta-1)(\zeta-z)} d \zeta \wedge d \bar{\zeta}, \\
& h_{2}(z)=\frac{z(z-1)}{2 \pi i} \iint_{c} \frac{\mu(\zeta)(1-\chi(\zeta))}{\zeta(\zeta-1)(\zeta-z)} d \zeta \wedge d \bar{\zeta},
\end{aligned}
$$

where $\chi$ is the characteristic function of $\Delta^{\prime}$. Define cocycles $q \in Z^{1}(\Gamma, \Pi)$, $q_{1}, q_{2} \in Z^{1}\left(\Gamma^{\prime}, \Pi\right)$ by

$$
q(\gamma)=\gamma_{-1}^{*} h-h, \quad q_{j}(\gamma)=\gamma_{-1}^{*} h_{j}-h_{j} \quad(j=1,2),
$$

then $\left[q_{1}\right] \in \beta_{\Gamma^{\prime}, \Delta^{\prime}}^{*}\left(M_{c a n}\left(\Delta^{\prime}, \Gamma^{\prime}\right)\right)$ and $\left[q_{2}\right] \in \alpha_{\Gamma^{\prime}, \Delta^{\prime}}\left(E_{b}\left(\Delta^{\prime}, \Gamma^{\prime}\right)\right) \quad$ (cf. [9, V, Theorems 2.4 and 4.2]), where $\left[q_{j}\right]$ denotes the cohomology class of $q_{j}(j=1,2)$. Moreover, we have $\left[\left.q\right|_{\Gamma^{\prime}}\right]=\left[q_{1}\right]+\left[q_{2}\right]$. Now, $J \circ \beta^{*}(\mu)=0$ implies $\left[\left.q\right|_{\Gamma^{\prime}}\right]=0$ and hence $\beta_{\Gamma^{\prime}, \Delta^{\prime}}^{*}(\mu \chi)=\left[q_{1}\right]=0$ (see (2.1)). Therefore, $\mu=0$ since the Bers map $\beta_{\Gamma^{\prime}, \Delta^{\prime}}^{*}: M_{c a n}\left(\Delta^{\prime}, \Gamma^{\prime}\right) \longrightarrow P H_{\Delta^{\prime}}^{1}\left(\Gamma^{\prime}, \Pi\right)$ is injective. q. e. d.

Ahlfors[1] defined two linear maps $\eta$ and $\delta$ having the following properties :

$$
\eta: \alpha(P E(\Delta, \Gamma)) \longrightarrow P H^{1}(G, \Pi),
$$

$(P E(\Delta, \Gamma)$ denotes the space of meromorphic parabolic Eichler integrals and $\alpha$ is the period map.) 
$\delta: A(\Delta, \Gamma) \longrightarrow P H^{1}(G, \Pi)$,

(2.3) $\operatorname{Im} \delta \cap J\left(H^{1}(\Gamma, \Pi)\right) \subset J \circ \alpha\left(E_{b}(4, \Gamma)\right)$ (cf. [1, Lemma 7]).

Furthermore, there is a direct decomposition

(2.4) $P H^{1}(G, \Pi)=\operatorname{Im} \eta+\operatorname{Im} \delta \quad$ (cf. [1, Lemma 8 and its proof $]$ ).

Lemma 2. $J \circ \beta^{*}\left(M_{c a n}(\Delta, \Gamma)\right) \cap \operatorname{Im} \delta=\{0\}$.

Proof. It follows from (2.3) that

$$
\begin{aligned}
& J \circ \beta^{*}\left(M_{c a n}(\Delta, \Gamma)\right) \cap \operatorname{Im} \delta \\
= & J \circ \beta^{*}\left(M_{c a n}(\Delta, \Gamma)\right) \cap J\left(H^{1}(\Gamma, \Pi)\right) \cap \operatorname{Im} \delta \\
\subset & J \circ \beta^{*}\left(M_{c a n}(\Delta, \Gamma)\right) \cap J \circ \alpha\left(E_{b}(\Delta, \Gamma)\right) .
\end{aligned}
$$

If $J \supset \beta^{*}(\mu) \in \operatorname{Im} \delta$ with $\mu \in M_{c a n}(\Delta, \Gamma)$, then there is some $F \in E_{b}(\Delta, \Gamma)$ such that $J \circ \beta^{*}(\mu)=J \circ \alpha(F)$. Let $q, q_{1}, q_{2}$ be cocycles as in the proof of Lemma 1. Then $J \circ \beta^{*}(\mu)=J \circ \alpha(F)$ implies $\left[q_{1}\right]+\left[q_{2}\right]=\left[\left.q\right|_{\Gamma^{\prime}}\right]=\alpha\left(\left.F\right|_{\Lambda^{\prime}}\right)$ and so $\left[q_{1}\right]=0$ (see (2.1)). Hence, $\mu=0$.

q. e. d.

Theorem 2. Under the same assumption as in Theorem 1 , we have a direct decomposition

$$
P H^{1}(G, \Pi)=J \circ \beta^{*}\left(M_{c a n}(\Delta, \Gamma)\right)+\operatorname{Im} \delta .
$$

Proof. From Lemma 1, Lemma 2, (2.2) and (2.4), one can easily prove the theorem. q. e. d.

For later use, we give the definition of $\delta$. For each $\varphi \in A(4, \Gamma)$ and $c \in \pi_{1}\left(S_{0}, p_{0}\right)$, set

$$
v_{\varphi}(c N)(z)=\int_{\left(c^{-1}\right)^{\prime}}(z-\zeta)^{2} \varphi(\zeta) d \zeta,
$$

where $\left(c^{-1}\right)^{\prime}$ denotes the lift of $c^{-1}$ with the initial point $\zeta_{0}$. Then, $v_{\varphi}(c N) \in \Pi$ and $v_{\varphi} \in Z^{1}(G, \Pi) . \delta(\varphi)$ is the cohomology class determined by $v_{\varphi}$.

\section{§ 3. Homomorphisms.}

(1) Spaces of homomorphisms.

Let $\Gamma$ be a Kleinian group generated by finitely many elements $\gamma_{1}, \ldots, \gamma_{r}$, and let $\Delta$ be an invariant union of components of $\Gamma$. We denote by $\operatorname{Hom}(\Gamma, M \ddot{o} b)$ the set of all homomorphisms from $\Gamma$ into $M \ddot{b} b$. Let $\chi \in \operatorname{Hom}(\Gamma, M \ddot{o} b)$. If $\operatorname{trace}^{2} \chi(\gamma)=4$ whenever $\gamma \in \Gamma$ is a parabolic element determining a puncture of $\Delta / \Gamma$, then $\chi$ is called $\Delta$-parabolic. We denote by $\operatorname{Hom}_{\Delta-p}(\Gamma, M \ddot{\prime} b)$ the set of all $\Delta$-parabolic homomorphisms. If there is a quasiconformal automorphism $w: \hat{\boldsymbol{C}} \longrightarrow \hat{\boldsymbol{C}}$ such that $\chi(\gamma)=$ worow $w^{-1}$ for all $\gamma \in \Gamma$, then $\chi$ is said to be a quasiconformal deformation of 
$\Gamma$, and the set of all such homomorphisms will be denoted by Hom $_{q c}$ $(\Gamma, M \ddot{b})$.

By the correspondence $\operatorname{Hom}(\Gamma, M \ddot{o} b) \ni \chi \longleftrightarrow\left(\chi\left(\gamma_{1}\right), \ldots, \chi\left(\gamma_{r}\right)\right) \in(M \ddot{o} b)^{r}$ we can regard $\operatorname{Hom}(\Gamma, M \ddot{b} b)$ as a subset of $(M \ddot{o} b)^{r}(\mathrm{cf} . \S 1)$.

\section{(2) Quasiconformal deformations.}

Let $\Gamma$ be as in (1), and let $\Omega$ denote the set of discontinuity of $\Gamma$. For each $\mu \in M(\Omega, \Gamma)_{1}$, there is a unique quasiconformal automorphism $w^{\mu}$ of $\hat{\boldsymbol{C}}$ that fixes $0,1, \infty$ and satisfies the Beltrami equation $\frac{\partial w}{\partial \bar{z}}=\mu \frac{\partial w}{\partial z}$. $w^{\mu}$ is compatible with $\Gamma$, that is, $w^{\mu} \circ \gamma \circ\left(w^{\mu}\right)^{-1} \in M o ̈ b$ for all $\gamma \in \Gamma$. As is well known, $w^{\mu}$ depends holomorphically on $\dot{\mu}$.

We define a holomorphic map $\Phi_{\Gamma}: M \ddot{o} b \times M(\Omega, \Gamma)_{1} \longrightarrow \operatorname{Hom}_{q c}(\Gamma, M \ddot{b} b)$ by $\Phi_{\Gamma}(g, \mu)(\gamma)=\left(g \circ w^{\mu}\right) \circ \gamma \circ\left(g \circ w^{\mu}\right)^{-1} \quad(\gamma \in \Gamma)$.

\section{(3) Schwarzian derivaitves.}

We assume the hypothesis of Theorem 1, and use the same notation.

Choose a disk $U_{0}$ about $\zeta_{0}$ such that $\gamma\left(U_{0}\right) \quad(\gamma \in \Gamma)$ are mutually disjoint. For every $\varphi \in A(\Delta, \Gamma)$, we denote by $W_{\varphi}$ the function element about $\zeta_{0}$ satisfying the Schwarzian differential equation

$$
S W_{\varphi}=\left[\frac{W_{\varphi}^{\prime \prime}}{W_{\varphi}^{\prime}}\right]^{\prime}-\frac{1}{2}\left[\frac{W_{\varphi}^{\prime \prime}}{W_{\varphi}^{\prime}}\right]^{2}=\varphi
$$

and the normalization condition

$$
W_{\varphi}(z)=z+O\left(\left|z-\zeta_{0}\right|^{3}\right) \quad\left(z \longrightarrow \zeta_{0}\right) .
$$

(We have assumed that $\zeta_{0} \neq \infty$.)

Let $c N \in G$. Denote by $W_{\varphi}^{\left(c^{-1}\right)^{\prime}}$ the function element about $\sigma(c N)^{-1}\left(\zeta_{0}\right)$ obtained from $W_{\varphi}$ by the analytic continuation along the curve $\left(c^{-1}\right)^{\prime}$, the lift of $c^{-1}$ with the initial point $\zeta_{0}$. Then there is some $\Psi(\varphi)(c N) \in$ Möb such that

$$
W_{\varphi} \circ \sigma(c N)=\Psi(\varphi)(c N) \circ W_{\varphi}^{\left(c^{-1}\right)^{\prime}}
$$

on $\sigma(c N)^{-1}\left(U_{0}\right)$. Then, it is easy to see that $\Psi(\varphi) \in \operatorname{Hom}(G, M \ddot{b} b)$. The next lemma follows from similar argument employed by Gardiner-Kra [5, Theorem 7.2]

Lemma 3. The mapping $\Psi: A(\Delta, \Gamma) \ni \varphi \longmapsto \Psi(\varphi) \in \operatorname{Hom}_{p}(G, M \ddot{b} b)$ is holomorphic and injective.

Proof. Since solutions of linear differential equations depend holomorphically on parameters, $\varphi \longmapsto \Psi(\varphi)$ is clearly holomorphic.

Next, let $\Delta^{\prime}$ be the component of $\Delta$ that contains $\zeta_{0}$ and set $\Gamma^{\prime}=$ $\operatorname{stab}\left(\Delta^{\prime}\right)$. Let $h: U \longrightarrow \Delta^{\prime}$ be a holomorphic universal covering map, where $U$ denotes the upper half plane, and set 


$$
F=\left\{g \in M o ̈ b \mid g(U)=U, \text { and } h \circ g=\rho(g) \circ h \text { for some } \rho(g) \in \Gamma^{\prime}\right\} .
$$

$F$ is a finitely generated Fuchsian group of the first kind with $U / F=S$, and is isomorphic to $G . \rho: F \longrightarrow \Gamma^{\prime}$ defines a surjective homomorphism. ( $F$ is called the Fuchsian model of $\Gamma^{\prime}$ via $h_{\text {.) }}$

Fix $z_{0} \in h^{-1}\left(\zeta_{0}\right)$ and denote by $\tilde{W}_{\varphi}$ the one-valued meromorphic function on $U$ obtained from the function element $W_{\varphi} \circ h$ near $z_{0} . \tilde{W}_{\varphi}$ induces an element $\chi_{\varphi}$ of $\operatorname{Hom}(F, M o ̈ b)$. Since $S h \in A(U, F)$ by Kra [7, Theorem 2], $S \tilde{W}_{\varphi}=h_{2}^{*} \varphi+S h$ also belongs to $A(U, F)$, and hence $\chi_{\varphi} \in \operatorname{Hom}_{p}(F, M \ddot{o} b)$ (see, for example, [7, Lemma 1]).

Set $\tau=\sigma\left(U, F ; z_{0}\right)$ (see $\left.\S 1\right)$ and note that $\sigma=\rho \circ \tau$. If $g=\tau(c N) \quad(c N \in$ $G)$, then $\rho(g)=\sigma(c N)$ and in a neighborhood of $g^{-1}\left(z_{0}\right)$

$$
\begin{aligned}
\chi_{\varphi}(g) \circ \tilde{W}_{\varphi} & =\tilde{W}_{\varphi} \circ g=W_{\varphi} \circ h \circ g=W_{\varphi} \circ \rho(g) \circ h=W_{\varphi} \circ \sigma(c N) \circ h \\
& =\Psi(\varphi)(c N) \circ W_{\varphi}^{(c-1)^{\prime}} \circ h=\Psi(\varphi)(c N) \circ \tilde{W}_{\varphi} .
\end{aligned}
$$

Hence, $\chi_{\varphi}(g)=\Psi(\varphi)(c N)$. In particular, $\Psi(\varphi) \in \operatorname{Hom}_{p}(G, M \ddot{o} b)$. Moreover, if $\Psi\left(\varphi_{1}\right)=\Psi\left(\varphi_{2}\right)$, then $S \tilde{W}_{\varphi_{1}}=S \tilde{W}_{\varphi_{2}}$ by $\operatorname{Kra}[8]$ and we see that $\varphi_{1}=\varphi_{2}$.

q. e. d.

Note that $W_{\varphi}$ defines a one-valued meromorphic function on $U^{\prime}$ if and only if $\Psi(\varphi)$ induces an element of $\operatorname{Hom}_{\Delta^{\prime}-p}\left(\Gamma^{\prime}, M \ddot{b} b\right)$, where $\Delta^{\prime}$ and $\Gamma^{\prime}$ are as in the proof of Lemma 3.

We conclude this section by proving the following lemma.

Lemma 4. Let $\Delta^{\prime}$ be a component of a Kleinian group $\Gamma$ and set $\Gamma^{\prime}=$ $\operatorname{stab}\left(\Delta^{\prime}\right)$. Let $\chi \in \operatorname{Hom}(\Gamma, M o ̈ b)$. Suppose that there is a function $f$ on $\Delta^{\prime}$ such that $f \circ \gamma=\chi(\gamma) \circ f$ on $\Delta^{\prime}$ for all $\gamma \in \Gamma^{\prime}$. Then there is a function $F$ on $\Delta=\bigcup_{\gamma \in \Gamma} \gamma\left(\Delta^{\prime}\right)$ such that $\left.F\right|_{\Delta^{\prime}}=f$ and $F \circ \gamma=\chi(\gamma) \circ F$ on $\Delta$ for all $\gamma \in \Gamma$.

Proof. We set $F(z)=\chi(\gamma) \circ f \circ \gamma^{-1}(z)$ when $z \in \gamma\left(\Delta^{\prime}\right)$ with $\gamma \in \Gamma$. If $\gamma_{1}\left(\Delta^{\prime}\right)=\gamma_{2}\left(\Delta^{\prime}\right) \quad\left(\gamma_{1}, \gamma_{2} \in \Gamma\right)$ and $z=\gamma_{1}(\zeta) \in \gamma_{1}\left(\Delta^{\prime}\right)$, then, since $\gamma_{2}^{-1} \cap \gamma_{1} \in \Gamma^{\prime}$,

$$
\begin{aligned}
\chi\left(\gamma_{2}\right) \circ f \circ \gamma_{2}^{-1}(z) & =\chi\left(\gamma_{2}\right) \circ f \circ \gamma_{2}^{-1} \circ \gamma_{1}(\zeta) \\
& =\chi\left(\gamma_{2}\right) \circ \chi\left(\gamma_{2}^{-1} \circ \gamma_{1}\right) \circ f(\zeta) \\
& =\chi\left(\gamma_{1}\right) \circ f \circ \gamma_{1}^{-1}(z) .
\end{aligned}
$$

Thus $F$ is well defined. Clearly, $\left.F\right|_{\Delta^{\prime}}=f$. Also, for every $z \in \Delta$ and $\gamma \in \Gamma$, assuming that $z \in \gamma_{1}\left(U^{\prime}\right)$, we have

$$
\begin{aligned}
F \circ \gamma(z) & =\chi\left(\gamma \circ \gamma_{1}\right) \circ f \circ\left(\gamma \circ \gamma_{1}\right)^{-1}(\gamma(z)) \\
& =\chi(\gamma) \circ \chi\left(\gamma_{1}\right) \circ f \circ \gamma_{1}^{-1}(z) \\
& =\chi(\gamma) \circ F(z) .
\end{aligned}
$$

The lemma is proved. q. e. d.

Remark. In the above lemma, if $f$ is meromorphic, then so is $F$. 


\section{§4. Proof of Theorem 1 .}

Now, we will give the proof of Theorem 1. We shall use the same notation as in $\S 3,(3)$.

Let $F$ be the Fuchsian model of $\Gamma^{\prime}$ via a holomorphic universal covering map $h: U \longrightarrow \Delta^{\prime}$, where $U$ denotes the upper half plane (see the proof of Lemma 3). If $\mu \in M_{c a n}(\Delta, \Gamma)_{1}$, then $\nu=h_{-1,1}^{*} \mu \in M_{c a n}(U, F)_{1}$. Gardiner-Kra [5, §11] defined a homeomorphism $R_{0}(\mu): A(U, F) \longrightarrow$ $A\left(w^{\nu}(U), w^{\nu} \circ F_{\circ}\left(w^{\nu}\right)^{-1}\right)$ such that for each fixed $\tilde{\varphi} \in A(U, F), R_{0}(\mu)(\tilde{\varphi})$ depends holomorphically on $\mu$. Using this $R_{0}$, we define a homeomorphism $R(\mu): A(\Delta, \Gamma) \longrightarrow A\left(w^{\mu}(\Delta), w^{\mu} \circ \Gamma \circ\left(w^{\mu}\right)^{-1}\right)$ by $R(\mu)=\left(\left(h^{\mu}\right)_{2}^{*}\right)^{-1} \circ R_{0}(\mu) \circ h_{2}^{*}$, where $h^{\mu}=\left.w^{\mu} \circ h_{\circ}\left(w^{\nu}\right)^{-1}\right|_{w^{\nu}(U)}$. For each fixed $\varphi \in A(\Delta, \Gamma), R(\mu)(\varphi)$ depends holomorphically on $\mu$.

Let $c N \in G$. For $\varphi \in A(\Delta, \Gamma)$ and $\mu \in M_{c a n}(\Delta, \Gamma)_{1}$, let $W_{R(\mu)(\varphi)}^{\left.(c)^{-1}\right)^{\prime}}$ denote the function element about $w^{\mu} \circ \sigma(c N)^{-1}\left(\zeta_{0}\right)$ obtained from the function element $W_{R(\mu)(\varphi)}$, normalized at $w^{\mu}\left(\zeta_{0}\right)$, by the analytic continuation along the curve $w^{\mu}\left(\left(c^{-1}\right)^{\prime}\right)$ ( see $\left.\S 3,(3)\right)$. For any $g \in M o ̈ b$, there is some $f(g, \varphi, \mu)$ $(c N) \in M \ddot{o} b$ such that

$$
g \circ W_{R(\mu)(\varphi)} \circ w^{\mu} \circ \sigma(c N)=f(g, \varphi, \mu)(c N) \circ g \circ W_{R(\mu)(\varphi)}^{(c-1)} \circ w^{\mu}
$$

on $\sigma(c N)^{-1}\left(U_{0}\right)$. Thus we have defined a holomorphic map $f: M o ̈ b \times$ $A(\Delta, \Gamma) \times M_{c a n}(\Delta, \Gamma)_{1} \longrightarrow \operatorname{Hom}_{p}(G, M \ddot{b} b)$ such that $f(e, 0,0)=\sigma$. We fix generators $c_{1} N, \ldots, c_{r} N$ of $G$ and set $\gamma_{j}=\sigma\left(c_{j} N\right) \quad(j=1, \ldots, r)$. One can consider that the image of $f$ is contained in $(M \ddot{o} b)^{r}$.

Before showing that $f$ has the desired property, we must define one more cohomology space. Let $\mathrm{Ad}: g \longmapsto \mathrm{Ad}(\mathrm{g})$ be the adjoint representation of the Lie group $M \ddot{b} b$. We set simply $\operatorname{Ad}(g)(X)=X^{g}$ for $g \in M \ddot{b} b$ and $X \in g$, where $g$ is the Lie algebra of $M o ̈ b . g$ can be identified with the tangent space $T_{e}(M o ̈ b)$ to $M o ̈ b$ at $e$. $G$ acts on $g$ via $g \times G \ni(X, c N)$ $\longmapsto X^{o(c N)} \in g$. Hence we can define, as in $\S 2$, the cohomology space $H^{1}(G, g)$ and the parabolic cohomology space $P H^{1}(G, g)$. There is a canonical isomorphism $I: Z^{1}(G, g) \longrightarrow Z^{1}(G, \Pi)$ such that $I\left(P Z^{1}(G, g)\right)$ $=P Z^{1}(G, \Pi)$ (cf. $\left.[5, \S 6]\right)$.

Now, identifying the tangent spaces to $A(\Delta, \Gamma)$ and $M_{c a n}(\Delta, \Gamma)_{1}$ at 0 with $A(\Delta, \Gamma)$ and $M_{c a n}(\Delta, \Gamma)$ respectively, we will examine the linear mapping $d\left(L_{\left(r_{1}^{-1} \ldots, r_{r}^{-1}\right)} \circ f\right)(e, 0,0): g \times A(\Delta, \Gamma) \times M_{c a n}(\Delta, \Gamma) \longrightarrow g^{r}$, where $L_{\left(g_{1}, \ldots, g_{r}\right)}$ denotes the left translation of the Lie group $(M \ddot{b} b)^{r}$.

For fixed $\varphi \in A(\Delta, \Gamma)$, the $\operatorname{map} p_{\varphi}: G \longrightarrow g$,

$$
p_{\varphi}(c N)=\lim _{t \rightarrow 0} \frac{\sigma(c N)^{-1} \circ f(e, t \varphi, 0)(c N)-e}{t},
$$

defines a cocycle. Using the isomorphism $I$ and setting $\gamma=\sigma(c N)$, we have, for $z \in \gamma^{-1}\left(U_{0}\right)$, 


$$
\begin{aligned}
& I\left(p_{\varphi}\right)(c N)(z) \\
= & \frac{1}{2} \int_{\zeta_{0}}^{r(z)} \frac{(\gamma(z)-\zeta)^{2}}{\gamma^{\prime}(z)} \varphi(\zeta) d \zeta-\frac{1}{2} \int_{\zeta_{0} \cdot\left(c^{-1}\right)^{\prime}}^{z}(z-\zeta)^{2} \varphi(\zeta) d \zeta \\
= & \frac{1}{2} \int_{r^{-1}\left(\zeta_{0}\right)}^{z}(z-\zeta)^{2} \varphi(\zeta) d \zeta-\frac{1}{2} \int_{\left.\zeta_{0^{\circ}(c}-1\right)^{\prime}}^{z}(z-\zeta)^{2} \varphi(\zeta) d \zeta \\
= & -\frac{1}{2} \int_{\zeta_{0^{\prime}}\left(c^{-1}\right)^{\prime}}^{\gamma^{-1}\left(\zeta_{0}\right)}(z-\zeta)^{2} \varphi(\zeta) d \zeta=-\frac{1}{2} v_{\varphi}(c N)(z) .
\end{aligned}
$$

Thus, the cohomology class of $I\left(p_{\varphi}\right)$ is $-\frac{1}{2} \delta(\varphi)$. Similarly, for fixed $\mu \in M_{c a n}(\Delta, \Gamma)$, the $\operatorname{map} q_{\mu}: G \longrightarrow g$,

$$
q_{\mu}(c N)=\lim _{t \rightarrow 0} \frac{\sigma(c N)^{-1} \circ f(e, 0, t \mu)(c N)-e}{t},
$$

also defines a cocycle. The cohomology class of $I\left(q_{\mu}\right)$ is $J \circ \beta^{*}(\mu)$. By the correspondence $Z^{1}(G, g) \ni z \longleftrightarrow\left(z\left(c_{1} N\right), \ldots, z\left(c_{r} N\right)\right) \in g^{r}$, we may regard $Z^{1}(G, g)$ as a subspace of $g^{r}$. Then, recalling Theorem 2, we see that

$$
M=\operatorname{Im} d\left(L_{\left(r_{1}^{-1} \ldots . r_{r}^{-1}\right)} \circ f\right)(e, 0,0)=P Z^{1}(G, g) .
$$

Let $\left\{w_{\alpha}\right\}_{\alpha \in A}$ be the set of the defining words of $G$. One can define a holomorphic map $F_{\alpha}:(M \ddot{o} b)^{r} \longrightarrow M \ddot{b} b$ by $F_{\alpha}\left(g_{1}, \ldots, g_{r}\right)=w_{\alpha}\left(g_{1}, \ldots, g_{r}\right)$ for each $\alpha \in A$. Then we have

$$
L_{A}=\underset{\alpha \in A}{\cap} \operatorname{Ker} d\left(F_{\alpha} \circ L_{\left(\gamma_{1}, \ldots, \gamma_{r}\right)}\right)(e, \ldots, e)=Z^{1}(G, g) .
$$

Also, let $w_{\beta}$ be a word in the generators that corresponds to a loop around a puncture of $S(\beta \in B)$, and define a holomorphic map $F_{\beta}$ : $(M \ddot{o} b)^{r} \longrightarrow \boldsymbol{C}$ by $F_{\beta}\left(g_{1}, \ldots, g_{r}\right)=\operatorname{trace}^{2} w_{\beta}\left(g_{1}, \ldots, g_{r}\right)$. Then, setting $L_{B}=$ $\bigcap_{\beta \in B} \operatorname{Ker} d\left(F_{\beta} \circ L_{\left(r_{1}, \ldots, r_{r}\right)}\right)(e, \ldots, e)$, we see that

$$
L=L_{A} \cap L_{B}=P Z^{1}(G, g) .
$$

It follows from (4.1) and (4.2) that $L=M$.

Clearly, we have

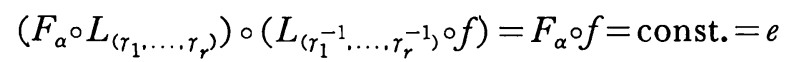

and

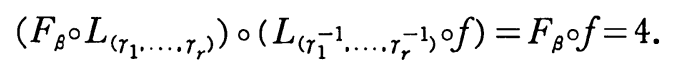

Consequently, by Weil's lemma [16, Lemma 1], there exists a neighborhood $E$ of $(e, 0,0)$ in $M o ̈ b \times A(\Delta, \Gamma) \times M_{c a n}(\Delta, \Gamma)_{1}$ and a neighborhood $\tilde{E}^{\prime}$ of $(e, \ldots, e)$ in $(M o ̈ b)^{r}$ such that

$$
L_{\left(\gamma_{1}^{-1}, \ldots, r_{r}^{-1}\right)} \circ f: E \longrightarrow
$$


is a biholomorphic homeomorphism. Since $L_{\left(r_{1} \ldots, r_{r}\right)}$ is a holomorphic bijection of $(M o ̈ b)^{r}$,

$$
f: E \longrightarrow\left\{\bigcap_{\alpha \in A} F_{\alpha}^{-1}(e)\right\} \cap\left\{\bigcap_{B \in B} F_{\beta}^{-1}(4)\right\} \cap E^{\prime}
$$

is also a biholomorphic homemorphism, where $E^{\prime}=L_{\left(\gamma_{1}, \ldots, r_{r}\right)}\left(\tilde{E}^{\prime}\right)$. Note that $\left\{\bigcap_{\alpha \in A} F_{\alpha}^{-1}(e)\right\} \cap\left\{\bigcap_{\beta \in B} F_{\beta}^{-1}(4)\right\}=\operatorname{Hom}_{p}(G, M \ddot{o} b)$ and that $E^{\prime}$ is a neighborhood of $\sigma=\left(\gamma_{1}, \ldots, \gamma_{r}\right)$ in $(M \ddot{o} b)^{r}$.

This completes the proof of Theorem 1.

The followings are corollaries to Theorem 1 .

Corollary 1. Let $\Delta^{\prime}$ be a component of a finitely generated (non-elementary) Kleinian group $\Gamma$ and set $\Gamma^{\prime}=\operatorname{stab}\left(\Delta^{\prime}\right)$. Let $F$ be the Fuchsian model of $\Gamma^{\prime}$ via a holomorphic universal covering map $h: U \longrightarrow \Delta^{\prime}$, where $U$ is the upper half plane. Let $\rho: F \longrightarrow \Gamma^{\prime}$ denote the canonical surjection, that is, hog= $\rho(g) \circ h$ for all $g \in F$. Then, $\operatorname{Hom}_{p}(F, M \ddot{o b})$ is a complex manifold of dimension $2 \operatorname{dim} A(U, F)+3$ in a neighborhood of $\rho$.

Proof. There is a bijective homomorphism $\tau: G \longrightarrow F$ such that $\rho \circ \tau$ $=\sigma$, where $G$ and $\sigma$ are as in Theorem 1. $\tau$ induces a homeomorphism $\tau^{*}: \operatorname{Hom}_{p}(F, M \ddot{o} b) \longrightarrow \operatorname{Hom}_{p}(G, M \ddot{o} b), \tau^{*}(\chi)=\chi \circ \tau$. Since $\tau^{*}(\rho)=\sigma$, the corollary follows at once from Theorem 1.

q. e. d.

Corollary 2. Let $\Gamma$ be a (non-elementary) Kleinian group generated by finitely many elements $\gamma_{1}, \ldots, \gamma_{r}$. Let $\Delta$ be an invariant union of components of $\Gamma$. Assume that $\Delta / \Gamma$ is a single Riemann surface. Fix $\zeta_{0} \in \Delta-\{$ elliptic fixed points $\}$ and denote by $W_{R(\mu)(\varphi)}$ the function element near $w^{\mu}\left(\zeta_{0}\right)$ that satisfies $S W_{R(\mu)(\varphi)}=R(\mu)(\varphi)$ and is normalized at $w^{\mu}\left(\zeta_{0}\right)$. Then there are neighborhoods $V$ of $(e, 0,0)$ in $M o ̈ b \times A(\Delta, \Gamma) \times M_{c a n}(\Delta, \Gamma)_{1}$ and $W$ of $\mathrm{id}=$ $\left(\gamma_{1}, \ldots, \gamma_{r}\right)$ in $(M o ̈ b)^{r}$ such that the following properties are valid:

For each $\chi \in \operatorname{Hom}_{\Delta-p}(\Gamma, M o ̈ b) \cap W$, there is a unique $(g, \varphi, \mu) \in V$ such that $W_{R(\mu)(\varphi)}$ determines a one-valued meromorphic function on $w^{\mu}(\Delta)$ and that

$$
g \circ W_{R(\mu)(\varphi)} \circ w^{\mu} \circ \gamma(z)=\chi(\gamma) \circ g \circ W_{R(\mu)(\varphi)} \circ w^{\mu}(z)
$$

for all $r \in \Gamma$ and $z \in \Delta$.

Proof. We shall use the same notation as in the proof of Theorem 1, except for $\gamma_{1}, \ldots, \gamma_{r} . \sigma$ induces a continuous map $\sigma^{*}: \operatorname{Hom}_{\Delta-p}(\Gamma, M \ddot{b} b)$ $\longrightarrow \operatorname{Hom}_{p}(G, M \ddot{o} b), \sigma^{*}(\chi)=\chi \circ \sigma$. There is a neighborhood $W$ of $\left(\gamma_{1}, \ldots\right.$, $\left.\gamma_{r}\right)$ in $(M \ddot{o} b)^{r}$ such that $\operatorname{Hom}_{\Delta-p}(\Gamma, M \ddot{o} b) \cap W \subset\left(\sigma^{*}\right)^{-1}\left(\operatorname{Hom}_{p}(G, M \ddot{o} b) \cap E^{\prime}\right)$. Take $V=E^{\prime}$. If $\chi \in \operatorname{Hom}_{\Delta-p}(\Gamma, M \ddot{o} b) \cap W$, then there is a unique element $(g, \varphi, \mu) \in V$ such that $W_{R(\mu)(\varphi)}$ determines a meromorphic function on $w^{\mu}\left(\Delta^{\prime}\right)$ and that (4.3) is true for all $\gamma \in \Gamma^{\prime}$ and $z \in \Delta^{\prime}$. By Lemma 4, $W_{R(\mu)(\varphi)}$ can be extended to $w^{\mu}\left(\Delta-\Delta^{\prime}\right)$ so that $(4.3)$ is also true for all $r \in \Gamma$ and $z \in \Delta$.

q. e. d. 
Remark. A homomorphism $\chi \in \operatorname{Hom}(\Gamma, M o ̈ b)$ is said to be allowable if $\operatorname{trace}^{2} \chi(\gamma)=\operatorname{trace}^{2} \gamma$ whenever $\gamma$ is elliptic or parabolic (cf. Bers [3] or [4]). From Corollary 2, one easily sees that if $\chi \in \operatorname{Hom}_{\Delta-p}(\Gamma, M \ddot{b} b) \cap V$, then $\operatorname{trace}^{2} \chi(\gamma)=\operatorname{trace}^{2} \gamma$ whenever $\gamma$ is an elliptic element with one of its fixed points in $\Delta$, or a parabolic element corresponding to a puncture of $\Delta / \Gamma$ (cf. [3, Lemma 3]).

\section{§ 5. Quasi-stability.}

Let $\Gamma$ be a Kleinian group generated by finitely many elements $\gamma_{1}$, $\ldots, \gamma_{r}$, and let $\Delta$ be an invariant union of components of $\Gamma$. We assume that $\Delta / \Gamma$ is a single Riemann surface. We fix $\zeta_{0} \in \Delta-\{$ elliptic fixed points $\}$ to define the normalized function element $W_{R(\mu)(\varphi)}$ near $w^{\mu}\left(\zeta_{0}\right) . \quad S(\Delta, \Gamma)$ (or $S_{q c}(\Delta, \Gamma)$ ) will denote the set of all pairs $(\varphi, \mu) \in A(\Delta, \Gamma) \times M_{c a n}$ $(\Delta, \Gamma)_{1}$ such that $W_{R(\mu)(\varphi)}$ defines a one-valued meromorphic function on $w^{\mu}(\Delta)$ and that $W_{R(\mu)(\varphi)} \circ w^{\mu}$ induces an element of $\operatorname{Hom}_{\Delta-p}(\Gamma, M \ddot{b} b)$ (or $\left.\operatorname{Hom}_{q c}(\Gamma, M \ddot{b} b)\right)$. This induced homomorphism is denoted by $T(\varphi$, $\mu)=T_{\Gamma \cdot \zeta_{0}}(\varphi, \mu)$. Note that $S(\Delta, \Gamma)$ (or $S_{q c}(\Delta, \Gamma)$ ) does not depend on $\zeta_{0}$.

Recall the definition of the map $\Phi_{\Gamma}$ (see $\left.\S 3,(2)\right) . \quad \Gamma$ is called quasistable if, for each neighborhood $E$ of 0 in $M(\Omega, \Gamma)_{1}$, one can find a neighborhood $E^{\prime}$ of $\mathrm{id}=\left(\gamma_{1}, \ldots, \gamma_{r}\right)$ in $(M \ddot{o} b)^{r}$ such that

$$
\operatorname{Hom}_{q c}(\Gamma, M \ddot{o} b) \cap E^{\prime} \subset \Phi_{\Gamma}(M \ddot{o} b \times E) .
$$

Theorem 3. Let $\Gamma$ be a finitely generated (non-elementary) Kleinian group and let $\Delta$ be an invariant union of components of $\Gamma$. Assume that $\Delta / \Gamma$ is a single Riemann surface. If $\Gamma$ is quasi-stable, then there is a neighborhood $B$ of $(0,0)$ in $A(\Delta, \Gamma) \times M_{c a n}(\Delta, \Gamma)_{1}$ such that for each $(\varphi, \mu) \in S_{q c}(\Delta, \Gamma) \cap B$, $W_{R(\mu)(\varphi)}$ is univalent on $w^{\mu}(\Delta)$ and has a quasiconformal extension to $\hat{\boldsymbol{C}}-w^{\mu}(\Delta)$ which is compatible with $w^{\mu} \circ \Gamma_{\circ}\left(w^{\mu}\right)^{-1}$. Furthermore, this extension can be chosen so that its complex dilatation belongs to $M_{c a n}\left(w^{\mu}(\Omega), w^{\mu} \circ \Gamma \circ\left(w^{\mu}\right)^{-1}\right)$, where $\Omega$ donotes the set of discontinuity of $\Gamma$.

Proof. Let $V$ and $W$ be as in Corollary 2 to Theorem 1. We fix a system $\left\{\gamma_{1}, \ldots, \gamma_{r}\right\}$ of generators of $\Gamma$.

Let $(g, \mu) \in M o ̈ b \times M_{c a n}(\Omega, \Gamma)_{1}$. We set $\mu^{\prime}=\mu$ on $\Delta$ and $\mu^{\prime}=0$ on $\hat{\boldsymbol{C}}-\Delta$. Define $\mu^{\prime \prime} \in M_{c a n}\left(w^{\mu^{\prime}}(\Omega), w^{\mu^{\prime}} \circ \Gamma \circ\left(w^{\mu^{\prime}}\right)^{-1}\right)_{1}$ so that $w^{\mu}=w^{\mu^{\prime \prime}} \circ w^{\mu^{\prime}}$. Explicitly, $\mu^{\prime \prime}=\left(\left(w^{\mu^{\prime}}\right)_{-1,1}^{*}\right)^{-1} \mu$ on $w^{\mu^{\prime}}(\Omega-\Delta)$ and $\mu^{\prime \prime}=0$ on $w^{\mu^{\prime}}(\Delta \cup \Lambda)$, where $\Lambda=\hat{\boldsymbol{C}}-\Omega$. Since $w^{\mu^{\prime \prime}}$ is meromorphic and univalent on $w^{\mu^{\prime}}(\Delta), S w^{\mu^{\prime \prime}} \in$ $A\left(w^{\mu^{\prime}}(\Delta), w^{\mu^{\prime}} \circ \Gamma_{\circ}\left(w^{\mu^{\prime}}\right)^{-1}\right)$ and its norm is bounded by some constant not depending on $\mu$ (cf. Beardon-Gehring [2, Theorem 1]). Setting $\varphi=$ $R\left(\mu^{\prime}\right)^{-1}\left(S w^{\mu^{\prime \prime}}\right)$, we define $\tilde{g} \in M o ̈ b$ so that $g \circ w^{u^{\prime \prime}}=\tilde{g} \circ W_{R\left(\mu^{\prime}\right)(\varphi)}$ on $w^{\mu^{\prime}}(\Delta)$. We have obtained a map $j:(g, \mu) \longmapsto\left(\tilde{g}, \varphi, \mu^{\prime}\right)$. To see that $j$ is continuous at $(e, 0)$, suppose that $\left(g_{n}, \mu_{n}\right) \longrightarrow(e, 0)(n \longrightarrow \infty)$ and set 
$j\left(g_{n}, \mu_{n}\right)=\left(\tilde{g}_{n}, \varphi_{n}, \mu_{n}^{\prime}\right) . \quad\left\{\varphi_{n}\right\}$ is a bounded sequence in $A(\Delta, \Gamma)$. If $\left\{\varphi_{n_{k}}\right\}$ is a subsequence converging to $\varphi$, then $W_{R\left(\mu^{\prime}{ }_{n_{k}}\right)^{\prime}\left(\varphi_{n_{k}}\right)} \longrightarrow W_{\varphi}$ uniformly near $\zeta_{0} \in \Delta$. But, since $g_{n} \circ w^{\mu_{n}^{\prime \prime}} \longrightarrow e$ uniformly near $\zeta_{0}, \varphi$ must be 0 . Thus $\varphi_{n}$ $\longrightarrow 0$ and hence $\tilde{g}_{n} \longrightarrow e$. This means that $j$ is continuous at $(e, 0)$, and so we can find a neighborhood $N_{1} \times N_{2}$ of $(e, 0)$ in $M \ddot{o} b \times M_{c a n}(\Omega, \Gamma)_{1}$ such that $j\left(N_{1} \times N_{2}\right) \subset V$.

Now the quasi-stability of $\Gamma$ implies that there is a neighborhood $W^{\prime}$ of id $=\left(\gamma_{1}, \ldots, \gamma_{r}\right) \in(M \ddot{o} b)^{r}$ such that $\operatorname{Hom}_{q c}(\Gamma, M \ddot{o} b) \cap W^{\prime} \subset \Phi_{\Gamma}\left(N_{1} \times N_{2}\right) \cap W$ (cf. Sakan [15, Lemma 2]). There is a neighborhood $V^{\prime}$ of $(e, 0,0)$ in $M \ddot{o} b \times A(\Delta, \Gamma) \times M_{c a n}(\Delta, \Gamma)_{1}$ so that $V^{\prime} \subset V$ and all homomorphisms of $\Gamma$ induced by some $(g, \varphi, \mu) \in V^{\prime}$ belong to $\operatorname{Hom}_{\Delta-p}(\Gamma, M \ddot{b} b) \cap W^{\prime}$. Let $B$ be the image of $V^{\prime}$ under the natural projection $M \ddot{o} b \times A(\Delta, \Gamma) \times M_{c a n}(\Delta$, $\Gamma)_{1} \longrightarrow A(\Delta, \Gamma) \times M_{c a n}(\Delta, \Gamma)_{1} . \quad B$ is a neighborhood of $(0,0) \in A(\Delta, \Gamma) \times$ $M_{c a n}(\Delta, \Gamma)_{1}$.

If $(\varphi, \mu) \in S_{q c}(\Delta, \Gamma) \cap B$, then there are some $\chi \in \operatorname{Hom}_{\Delta-p}(\Gamma, M \ddot{b} b) \cap W^{\prime}$, $g \in M \ddot{o} b$ and $(h, \nu) \in N_{1} \times N_{2}$ such that $(g, \varphi, \mu) \in V^{\prime}$ and that

and

$$
\begin{array}{ll}
g \circ W_{R(\mu)(\varphi)} \circ w^{\mu} \circ \gamma=\chi(\gamma) \circ g \circ W_{R(\mu)(\varphi)} \circ w^{\mu} & (\text { on } \Delta) \\
h \circ w^{\nu} \circ \gamma=\chi(\gamma) \circ h \circ w^{\nu} & \text { (on } \hat{\boldsymbol{C}})
\end{array}
$$

for all $r \in \Gamma$. On the other hand, $\left(\tilde{h}, \psi, \nu^{\prime}\right)=j(h, \nu) \in V$ and

$$
\tilde{h} \circ W_{R\left(\nu^{\prime}\right)(\phi)} \circ w^{\nu^{\prime}} \circ \gamma=\chi(\gamma) \circ \tilde{h}_{\circ} \circ W_{R\left(\nu^{\prime}\right)(\phi)} \circ w^{\nu^{\prime}} \quad(\text { on } \Delta)
$$

for all $r \in \Gamma$, where $W_{R\left(\nu^{\prime}\right)(\psi)}=\tilde{h}^{-1} \circ h \circ w^{\nu} \circ\left(w^{\nu^{\prime}}\right)^{-1}$ on $w^{\nu^{\prime}}(\Delta)$. Therefore, by uniqueness, $W_{R(\mu)(\varphi)}=\tilde{h}^{-1} \circ h \circ w^{\nu} \circ\left(w^{\nu^{\prime}}\right)^{-1}$ on $w^{\mu}(\Delta)$. q. e. d.

Remark. Bers conjectured in [4] that all finitely generated Kleinian groups should be quasi-stable. Kruškal' [10] answered to this conjecture in the affirmative. Unfortunately, however, it would seem hard to me to follow his argument.

\section{§6. Criteria for quasi-stability of finitely generated function groups.}

Theorem 4. Let $\Gamma$ be a finitely generated (non-elementary) Kleinian group with an invariant component $\Delta$. Set $T=T_{\Gamma, \zeta_{0}}$ with fixed $\zeta_{0} \in \Delta-\{$ elliptic fixed points\}. Then the following four conditions are equivalent to each other:

(i) $\Gamma$ is quasi-stable.

(ii) There is a neighborhood $B$ of $(0,0)$ in $A(\Delta, \Gamma) \times M_{c a n}(\Delta, \Gamma)_{1}$ such that $W_{R(\mu)(\varphi)} \circ w^{\mu}(\Delta) \neq \hat{\boldsymbol{C}}$ for each $(\varphi, \mu) \in S_{q c}(\Delta, \Gamma) \cap B$.

(iii) There is a neighborhood $B$ of $(0,0)$ in $A(\Delta, \Gamma) \times M_{c a n}(\Delta, \Gamma)_{1}$ such that $T(\varphi, \mu)(\Gamma)$ acts discontinuously on $W_{R(\mu)(\varphi)} \circ w^{\mu}(\Delta)$ for each $(\varphi, \mu) \in$ $S_{q c}(\Delta, \Gamma) \cap B$.

(iv) There is a neighborhood $B$ of $(0,0)$ in $A(\Delta, \Gamma) \times M_{c a n}(\Delta, \Gamma)_{1}$ such that for each $(\varphi, \mu) \in S_{q c}(\Delta, \Gamma) \cap B, W_{R(\mu)(\varphi)}$ is univalent on $w^{\mu}(\Delta)$ and has 
a quasiconformal extension to $\hat{\boldsymbol{C}}-w^{\mu}(\Delta)$ which is compatible with $w^{\mu} \circ \Gamma_{\circ}\left(w^{\mu}\right)^{-1}$.

Proof. Let $F$ be the Fuchsian model of $\Gamma$ via a holomorphic universal covering map $h: U \longrightarrow \Delta$, where $U$ denotes the upper half plane. For $\mu \in M(\Delta, \Gamma)_{1}$, we set $h_{\mu}=w^{\mu} \circ h_{\circ}\left(w_{\nu}\right)^{-1}, \Gamma_{\mu}=w^{\mu} \circ \Gamma_{\circ}\left(w^{\mu}\right)^{-1}, \Delta_{\mu}=w^{\mu}(\Delta)$ and $F_{\mu}=w_{\nu} \circ F_{\circ}\left(w_{\nu}\right)^{-1}$, where $\nu=h_{-1,1}^{*} \mu$ and $w_{\nu}$ is the unique quasiconformal automorphism of $U$, with the complex dilatation $\nu$, that fixes $0,1, \infty$. $h_{\mu}: U \longrightarrow \Delta_{\mu}$ is a holomorphic universal covering map and $F_{\mu}$ is the Fuchsian model of $\Gamma_{\mu}$ via $h_{\mu}$. We denote by $\rho$ and $\rho_{\mu}$ the canonical surjective homomorphisms $F \longrightarrow \Gamma$ and $F_{\mu} \longrightarrow \Gamma_{\mu}$, respectively.

( i ) $\Rightarrow$ (ii): This is a direct consequence of Theorem 3.

(ii) $\Leftrightarrow$ (iii) : Since $S\left(W_{R(\mu)(\varphi)} \circ h_{\mu}\right)=\left(h_{\mu}\right)_{2}^{*}(R(\mu)(\varphi))+S h_{\mu} \in A\left(U, F_{\mu}\right)$ for every $(\varphi, \mu) \in S(\Delta, \Gamma)$, it follows from $\operatorname{Kra}[7$, Theorem 1] that (ii) and (iii) are equivalent to each other.

(iii) $\Rightarrow$ (iv) : If $\Gamma$ is quasi-Fuchsian, then the result is clear since $\Gamma$ is quasi-stable.

Suppose that $\Gamma$ is not quasi-Fuchsian. Let $(\varphi, \mu) \in S_{q c}(\Delta, \Gamma) \cap B$. For convenience, we set $W=W_{R(\mu)(\varphi)}$. There are some $\chi \in \operatorname{Hom}_{q c}\left(\Gamma_{\mu}, M \ddot{b} b\right)$ and a quasiconformal automorphism $w$ of $\hat{\boldsymbol{C}}$ such that

$$
W \circ \gamma=\chi(\gamma) \circ W \quad \text { and } \quad w \circ \gamma=\chi(\gamma) \circ w
$$

for all $\gamma \in \Gamma_{\mu}$. It follows from the assumption and [7, Theorem 1] that $W\left(\Delta_{\mu}\right)$ and $w\left(\Delta_{\mu}\right)$ are invariant components of $\chi\left(\Gamma_{\mu}\right)=w \circ \Gamma_{\mu} \circ w^{-1}$. Thus, by Maskit [11, Theorem 2], $W\left(\Delta_{\mu}\right)=w\left(\Delta_{\mu}\right)$ because $\chi\left(\Gamma_{\mu}\right)$ is not quasiFuchisan.

Moreover, $W \circ h_{\mu}: U \longrightarrow W\left(\Delta_{\mu}\right)$ is a universal covering map (cf. [7, Theorem 1]). Let $\tilde{F}_{\mu}$ be the Fuchsian model of $\chi\left(\Gamma_{\mu}\right)$ via $W \circ h_{\mu}$. Clearly, $F_{\mu} \subset \tilde{F}_{\mu}$ since for every $g \in F_{\mu}, W \circ h_{\mu} \circ g=W \circ \rho_{\mu}(g) \circ h_{\mu}=\left(\chi \circ \rho_{\mu}(g)\right) \circ W \circ h_{\mu}$. On the other hand,

$$
\operatorname{Area}\left(U / \tilde{F}_{\mu}\right)=\operatorname{Area}\left(w\left(\Delta_{\mu}\right) / \chi\left(\Gamma_{\mu}\right)\right)=\operatorname{Area}\left(\Delta_{\mu} / \Gamma_{\mu}\right)=\operatorname{Area}\left(U / F_{\mu}\right),
$$

where Area $(S)$ denotes the Poincaré area of a Riemann surface $S$, so we must have $F_{\mu}=\tilde{F}_{\mu}$.

To show that $W$ is univalent, assume that $W\left(\zeta_{1}\right)=W\left(\zeta_{2}\right)=w_{0}$. We connect $\zeta_{1}$ with $\zeta_{2}$ by a curve $c$ in $\Delta_{\mu}$ and denote by $\tilde{c}$ its lift to $U$ via $h_{\mu}$ with the initial point $z_{1}$ and the end point $z_{2}$. Then $\tilde{c}$ is a lift of $W(c)$, a closed curve beginning at $w_{0}$, to $U$ via $W \circ h_{\mu}$, and hence there is some $g \in \tilde{F}_{\mu}=F_{\mu}$ with $g\left(z_{1}\right)=z_{2}$ such that $W \circ h_{\mu} \circ g=W \circ h_{\mu}$ on $U$. Since $W \circ h_{\mu}=W \circ h_{\mu} \circ g=W \circ \rho_{\mu}(g) \circ h_{\mu}=\left(\chi \circ \rho_{\mu}\right)(g) \circ W \circ h_{\mu}$, it follows that $\chi \circ \rho_{\mu}(g)=e$ and $\rho_{\mu}(g)=e$. Hence we have

$$
\zeta_{2}=h_{\mu}\left(z_{2}\right)=h_{\mu} \circ g\left(z_{1}\right)=\rho_{\mu}(g) \circ h_{\mu}\left(z_{1}\right)=h_{\mu}\left(z_{1}\right)=\zeta_{1} .
$$

Thus, $W$ is univalent.

We have shown that $w^{-1} \circ W: \Delta_{\mu} \longrightarrow \Delta_{\mu}$ is quasiconformal and that 
$w^{-1} \circ W \circ \gamma=\gamma \circ w^{-1} \circ W$ for all $\gamma \in \Gamma_{\mu}$. Therefore it follows from Maskit [12] that the function $u, u(z)=w^{-1} \circ W(z) \quad\left(z \in \Delta_{\mu}\right)$ and $u(z)=z\left(z \in \hat{\boldsymbol{C}}-\Delta_{\mu}\right)$, is a quasiconformal automorphism of $\hat{\boldsymbol{C}}$. wou is a quasiconformal extension of $W$ and is clearly compatible with $\Gamma_{\mu}$.

(iv) $\Rightarrow$ (i ) : Let $\left\{\Delta_{i}\right\}_{i=0}^{k}$ be a complete list of non-conjugate components of $\Gamma$ with $\Delta_{0}=\Delta$ ( $k$ may be 0 ) and let $\Gamma_{i}=\operatorname{stab}\left(\Delta_{i}\right)$ be generated by $\gamma_{1}^{i}, \ldots, \gamma_{n(i)}^{i}(i=0, \ldots, k)$. Note that $\Gamma_{0}=\Gamma$. Since $\bigcup_{i=0}^{k}\left\{\gamma_{j}^{i}\right\}_{j=1}^{n(i)}$ is a system of generators of $\Gamma$, we may consider that

$$
\operatorname{Hom}(\Gamma, M \ddot{o} b) \subset(M \ddot{o} b)^{n(0)} \times \ldots \times(M \ddot{o} b)^{n(k)}=(M \ddot{o} b)^{N} .
$$

For $i=0, \ldots, k$, let $p_{i}:(M \ddot{o} b)^{N} \longrightarrow(M \ddot{o} b)^{n(i)}$ denote the natural projection and let $\Omega_{i}$ be the set of discontinuity of $\Gamma_{i}$. For $0<t<1$, we set

and

$$
\begin{aligned}
& N_{i}(t)=\left\{\mu \in M_{c a n}\left(\Omega_{i}, \Gamma_{i}\right)_{1} \mid\|\mu\|_{\infty}<t\right\} \quad(i=0, \ldots, k) \\
& N^{\prime}(t)=M_{c a n}(\Delta, \Gamma)_{1} \cap N_{0}(t) .
\end{aligned}
$$

Next, let $V=V_{1} \times V_{2} \times V_{3}$ and $W$ be as in Corollary 2 to Theorem 1 . We may assume that $V_{2} \times V_{3} \subset B$ and $V_{3} \subset N^{\prime}(t)$. Moreover, we can assume that $W$ is so small that for $i=1, \ldots, k$,

$$
p_{i}(W) \cap \operatorname{Hom}_{q c}\left(\Gamma_{i}, M \ddot{o} b\right) \subset \Phi_{\Gamma_{i}}\left(V_{1} \times N_{i}(t)\right),
$$

since each $\Gamma_{i}(i \neq 0)$ is quasi-Fuchsian and hence quasi-stable.

If $\chi \in \operatorname{Hom}_{q c}(\Gamma, M \ddot{o b}) \cap W$, then there is some $(g, \varphi, \mu) \in V$ such that

$$
g \circ W_{R(\mu)(\varphi)} \circ w^{\mu} \circ \gamma=\chi(\gamma) \circ g \circ W_{R(\mu)(\varphi)} \circ w^{\mu}
$$

for all $\gamma \in \Gamma$. Set $w=g \circ W_{R(\mu)(\varphi)} \circ w^{\mu}$. The assumption implies that $w$ has a quasiconformal extension which is compatible with $\Gamma$. We denote it by the same letter $w$.

Since $\left.\chi\right|_{\Gamma_{i}} \in p_{i}(W) \cap \operatorname{Hom}_{q c}\left(\Gamma_{i}, M \ddot{o} b\right) \subset \Phi_{\Gamma_{i}}\left(V_{1} \times N_{i}(t)\right)$ for $i=1, \ldots, k$, there is a quasiconformal automorphism $f_{i}$ of $\hat{\boldsymbol{C}}$ with the complex dilatation $\mu_{i} \in N_{i}(t)$ such that $\chi(\gamma)=f_{i} \circ \gamma \circ f_{i}^{-1}$ for all $\gamma \in \Gamma_{i}$. Because $w^{-1} \circ f_{i} \circ \gamma$ $=\gamma \circ w^{-1} \circ f_{i}$ for all $\gamma \in \Gamma_{i}$, we have $w\left(\Delta_{i}\right)=f_{i}\left(\Delta_{i}\right)$ (cf. [12, Corollary 2]). Set $u(z)=w(z) \quad(z \in \Delta)$ and $u(z)=\chi(\gamma) \circ f_{i} \circ \gamma^{-1}(z) \quad\left(z \in \gamma\left(\Delta_{i}\right), \gamma \in \Gamma\right)$. Then $u$ is well defined (cf. the proof of Lemma 4). Furthermore, we have $w^{-1} \circ u\left(\Omega_{0}\right)=\Omega_{0}$ and $w^{-1} \circ u \circ \gamma=\gamma \circ w^{-1} \circ u$ for all $\gamma \in \Gamma$, for,

$$
u\left(\gamma\left(\Delta_{i}\right)\right)=\chi(\gamma) \circ f_{i}\left(\Delta_{i}\right)=\chi(\gamma) \circ w\left(\Delta_{i}\right)=w\left(\gamma\left(\Delta_{i}\right)\right)
$$

and $\quad u \circ \gamma(z)=\chi\left(\gamma \circ \gamma_{1}\right) \circ f_{i} \circ \gamma_{1}^{-1}(z)=w \circ \gamma_{\circ} w^{-1} \circ u(z) \quad\left(z \in \gamma_{1}\left(\Delta_{i}\right)\right)$.

Thus, it follows from [12] that $u$ can extend to a quasiconformal automorphism of $\hat{\boldsymbol{C}}$ if we set $u=w$ on $\hat{\boldsymbol{C}}-\Omega_{0}$. The complex dilatation of $u$ is in $N_{0}(t)$ and $u \circ \gamma \circ u^{-1}=w \circ \gamma \circ w^{-1}=\chi(\gamma) \quad(\gamma \in \Gamma)$. Hence, we have $\chi \in$ $\Phi_{\Gamma}\left(M \ddot{o} b \times N_{0}(t)\right)$. Thus $\operatorname{Hom}_{q c}(\Gamma, M \ddot{o} b) \cap W \subset \Phi_{\Gamma}\left(M \ddot{o} b \times N_{0}(t)\right)$ and so $\Gamma$ is quasi-stable.

q. e. d. 

[14].

Remark 1. The part (iv) $\Rightarrow$ (i) of the above proof is due to Sakan

Remark 2. Let $\Gamma$ be as in Theorem 4. If $\Gamma$ is quasiconformally stable, then by Nakada [13, Theorem 3] and Sakan [15, Theorem 1] $\Gamma$ is also quasi-stable. (For terminology used here, see, for example, [15]). In this case, one can choose a neighborhood $B$ of $(0,0)$ in $A(\Delta, \Gamma) \times$ $M_{c a n}(\Delta, \Gamma)_{1}$ such that if $(\varphi, \mu) \in S(\Delta, \Gamma) \cap B$ and $T(\varphi, \mu)$ is parabolic, then $W_{R(\mu)(\varphi)}$ is univalent and can extend to a quasiconformal automorphism of $\hat{\boldsymbol{C}}$. The proof is similar to that of Theorem 3 .

\section{DEPARTMENT OF MATHEMATICS KYOTO UNIVERSITY}

\section{References}

[1] L. V. Ahlfors, The structure of a finitely generated Kleinian group, Acta Math., 122 (1969), 1-17.

[2] A. F. Beardon and F. W. Gehring, Schwarzian derivatives, the Poincare metric and the kernel function, Comment. Math. Helv., 55 (1980), 50-64.

[3] L. Bers, On boundaries of Teichmüller spaces and on kleinian groups: I, Ann. of Math., 91 (1970), 570-600.

[4] L. Bers, Spaces of Kleinian groups, Lecture Notes in Mathematics, 155 (1970), Springer, Berlin, 9-34.

[5] F. Gardiner and I. Kra, Stability of Kleinian groups, Indiana Univ. Math. J., 21 (1972), 1037-1059.

[6] I. Kra, On cohomology of kleinian groups: II, Ann. of Math., 90 (1969) 576-590.

[7] I. Kra, Deformations of Fuchsian groups, II, Duke Math. J., 38 (1971), 499-508.

[8] I. Kra, A generalization of a theorem of Poincaré, Proc. Amer. Math. Soc., 27 (1971), 299-302.

[9] I. Kra, Automorphic Forms and Kleinian Groups, W. A. Benjamin, Reading, Massachusetts, 1972.

[10] S. L. Kruškal', Stability of Kleinian groups, Soviet Math. Dokl., 15 (1974), 822-825.

[11] B. Maskit, On boundaries of Teichmüller spaces and on kleinian groups: II, Ann. of Math., 91 (1970), 607-639.

[12] B. Maskit, Self-maps on Kleinian groups, Amer. J. Math., 93 (1971), 840-856.

[13] M. Nakada, Quasi-conformal stability of finitely generated function groups, Tôhoku Math. J., 30 (1978), 45-58.

[14] K. Sakan, On stability of Kleinian groups (Japanese), RIMS Kokyuroku, 318 (1978), 63-89.

[15] K. Sakan, On stability of finitely generated Kleinian groups, Osaka J. Math., 17 (1980), 165-176.

[16] A. Weil, Remarks on the cohomology of groups, Ann. of Math., 80 (1964), 149-157.

Current AdDress

DEPARTMENT OF MATHEMATICS

FACULTY OF SCIENCE

HIROSHIMA UNIVERSITY 